\title{
INFLUÊNCIA DA OXIDAÇÃO TÉRMICA SOBRE AS PROPRIEDADES DA LIGA TI 6AL 4V PARA APLICAÇÕES EM PRÓTESES - TRATAMENTO TÉRMICO DE 5 HORAS EM AR AMBIENTE
}

Eliane Sell Espindola ${ }^{1}$

\section{RESUMO}

As ligas de titânio se destacam entre os materiais para utilização em próteses por apresentarem elevada relação resistência mecânica/peso, boa resistência à corrosão e elevada biocompatibilidade. Dentre as ligas à base de titânio para aplicações biomédicas, a principal é a Ti-6Al-4V. No entanto, pesquisas recentes mostram que a dissolução de Al e $\mathrm{V}$ podem causar efeitos citotóxicos. Para limitar a dissolução desses elementos optou-se pela formação de uma camada de óxido por oxidação térmica. Esse tratamento também teve por objetivo aumentar a rugosidade superficial a fim de melhorar a osseo-integração em caso de implantes. Portanto, este estudo consiste em analisar a influência da oxidação térmica sobre as propriedades de superfície da liga Ti-6Al-4V. Os ensaios de oxidação foram realizados entre 300 e $700{ }^{\circ} \mathrm{C}$ em atmosfera de ar ambiente durante 5 horas. Os óxidos formados foram avaliados por ensaios de difração de raios $\mathrm{X}$, microscopia eletrônica de varredura, microdureza Vickers, aderência por dureza Rockwell-C, rugosidade superficial e corrosão-desgaste. Foi observado que a rugosidade superficial aumenta com a temperatura de oxidação. Por outro lado, a microdureza e a resistência à corrosão - desgaste atingem os melhores resultados nas amostras oxidadas a 600 e $650^{\circ} \mathrm{C}$, respectivamente. Os testes de aderência mostraram que o filme de óxido tem boa aderência a $600^{\circ} \mathrm{C}$. Acima dessa temperatura o filme de óxido tem baixa aderência devido à presença de maiores tensões no filme durante a sua formação.

Palavras-chave: Ti-6Al-4V. Oxidação. Corrosão. Desgaste. Biomateriais.

${ }^{1}$ Mestre; e-mail: eliane.espindola@sc.senai.br 


\section{INTRODUÇÃO}

O titânio (Ti) foi descoberto através o mineral ilmenita (FeTiO3), porém também foi identificado mais tarde pelo alemão Heinrich Klaproth no mineral rutilo (TiO2). Foi encontrado também no mineral anatásia (TiO2). Ambos de mesma composição, vem a diferenciar-se pela fase em que se apresentam (ZIMMER, 2006).

A liga comercial Ti-6Al-4V surgiu frente à necessidade de se alterar as propriedades mecânicas do titânio, visto que apesar de sua ótima resistência à corrosão, não apresenta propriedades mecânicas e tribológicas ideais para próteses sujeitas a elevados esforços mecânicos.Desta forma é um dos poucos materiais sobre o qual ocorre osseointegração após o seu implante (SUL, 2003). Existem dois tipos de resposta após o implante de um biomaterial (HENCH; WILSON, 1993) - o primeiro tipo consiste na formação de um tecido fibroso sobre o implante. Esta membrana de tecido fibroso não garante uma adequada fixação biomecânica com o osso, podendo soltar durante os esforços mecânicos. O segundo tipo de resposta está relacionado com o contato direto entre o osso e o implante sem a presença de uma membrana fibrosa. Esse tipo de resposta é conhecido por osseointegração. A liga Ti-6Al-4V possui composição química em peso: 5.5-6.7\%Al, 3.5-4.5\%V, e o restante em Ti (BATISTA AVELAR et al., 2006). Quanto ás propriedades mecânicas, a liga comercial Ti-6Al-4V é do tipo $a+b$, possui tensão de escoamento entre 825 e 869 MPa, resistência á tração entre 895 e $930 \mathrm{MPa}$, alongamento entre 6 e 10\% e módulo de elasticidade entre 110 e 114 GPa (MELLO ROCHA et al., 2004). Segundo Niinomi (1998), os valores considerados satisfatórios para aplicações cirúrgicas estão entre 500 e $1000 \mathrm{MPa}$. A interação biológica ocorre porque o óxido de titânio formado sobre o material é bio-inerte e atua como uma barreira eletroquímica contra a dissolução iônica (OKAZAKI; GOTOH, 2005), permitindo a aderência direta das células ósseas sobre a superfície de óxido, sem a formação de uma membrana de tecido ósseo (SUL, 2003; SUL et al., 2001; THOMSEN et al., 1997). A osseointegração e a proliferação das células entre o implante (filme de óxido) e o osso, depende da morfologia (rugosidade superficial) e composição da camada de óxido (LI et al., 2002; WENNEMBERG et al., 1998). Nas literaturas encontram-se várias publicações que afirmam que a rugosidade superficial do titânio e suas ligas têm influência na velocidade de osseointegração e na fixação biomecânica (COCHRAN et al., 1998; WENNERBERG et al., 1998). Também uma fixação rápida, com estabilidade mecânica em longo prazo faz o implante depender de uma elevada rugosidade superficial (BUSER et al., 1991; GOTFREDSEN et al., 1995; WENNERBERG et al., 1996). Superfícies anodizadas (oxidação anódica), cujo objetivo é aumentar a espessura de óxido, aumentam a aderência do osso sobre o implante quando comparado com o material não oxidado (SUL et al., 2002; ROCCl; MARTIGNONI; GOTLLOW, 2003). Os efeitos benéficos proporcionados pela oxidação anódica também podem ser obtidos pela oxidação térmica. Neste caso, estruturas, espessura e morfologia podem ser obtidas através do controle da temperatura e tempo de oxidação. Os ensaios de oxidação foram então realizados entre $300^{\circ} \mathrm{C}$ e $700^{\circ} \mathrm{C}$, em ar ambiente, por 5 horas. Os filmes de óxido foram caracterizados por microdureza Vickers, rugosidade superficial (Parâmetro Ra), aderência por Rockwell-C e testes de corrosão-desgaste com fluídos 
corpóreos simulados, através de ensaio pino-sobre-disco (ASTM G99). Porém estes últimos testes ainda estão em desenvolvimento.

O presente artigo apresenta como objetivo geral a Investigação das características do filme de óxido em uma faixa significativa de temperatura. E seus objetivos específicos: Avaliação da rugosidade superficial, microdureza e aderência do filme formado após oxidação térmica por 5 horas em ar ambiente, bem como avaliação de comportamento tribológico em testes de corrosão-desgaste com fluído corpóreo simulado.

\section{MATERIAIS E MÉTODOS}

Amostras da liga comercial Ti-6Al-4V foram lixadas com lixas de carbeto de silício e polidas até $1 \mathrm{~mm}$ com pasta diamantada. Posteriormente foram limpas em cuba de ultrassom, com água deionizada, lavadas com etanol e secadas a temperatura ambiente. Efetuou-se então ensaios de microdureza Vickers e rugosidade superficial Ra, de modo a conhecer os valores para após a oxidação térmica efetuar novos ensaios e análises comparativas.

As amostras foram oxidadas, individualmente, em forno tipo mufla, por um período de 5 horas e temperaturas de $300^{\circ} \mathrm{C}, 400^{\circ} \mathrm{C}, 500^{\circ} \mathrm{C}, 600^{\circ} \mathrm{C}, 650^{\circ} \mathrm{C}$ e $700^{\circ} \mathrm{C}$. O resfriamento ocorreu lentamente, dentro do forno; pois um resfriamento rápido poderia resultar em trincas no filme oxidado. A caracterização dos filmes de óxido formados foi efetuada através de ensaios de raio $x$ (DRX), microscopia eletrônica de varredura (MEV) e rugosidade superficial (Parâmetro Ra). Para identificar os óxidos de titânio formados, utilizou-se um difratômetro Shimadzu XRD-6000, com fonte de radiação Cua e incidência de $2^{\circ} / \mathrm{min}$; ângulo de difração compreendido entre $10^{\circ}$ e $80^{\circ}$; incremento de $0,02^{\circ}$ e tempo de 6 segundos. Para avaliar a rugosidade superficial, utilizou-se um rugosímetro digital Mitutoyo - Surftest 211, e foram efetuadas dez medidas aleatórias em cada amostra. O parâmetro de rugosidade utilizado foi o $\mathrm{Ra}$, cujos valores definem a média aritmética do perfil de superfície através de uma linha média. Os testes de microdureza foram efetuados com penetrador piramidal tipo Vickers, em equipamento Shimadzu HMV-2T, sob cargas de $98,01 \mathrm{mN}$ (equivalente a 10 gramas) e $980,1 \mathrm{mN}$ (equivalente a 100 gramas). Foram efetuadas dez medidas aleatórias em cada amostra. Um teste de dureza Rockwell-C foi utilizado para avaliar a aderência. Este teste utiliza um penetrador padrão de Rockwell-C, com carga de $150 \mathrm{Kg}$, de modo a romper o filme após a penetração. $\mathrm{O}$ rompimento do filme de óxido foi comparado a classes de aderência, a nível qualitativo (HEINKE et al., 1995).

As classes de aderência, que compreendem HF 1 a HF 4 são designados como sendo de boa aderência, enquanto as classes HF 5 e HF 6 representam modelos de baixa aderência. Em cada amostra foram efetuadas três impressões por penetração. Posteriormente, foi utilizado o equipamento de MEV marca Zeiss 940A, para avaliar as impressões efetuadas pela penetração de Rockwell-C e a morfologia de superfície das amostras oxidadas. 


\section{RESULTADOS E DISCUSSÕES}

\subsection{Difração de Raios X}

A figura 1 mostra os espectros das superfícies oxidadas, avaliados através de difração por raio x. A análise de DRX na amostra de $300^{\circ} \mathrm{C}$ apontou um filme cristalino de anatásia entre dois picos de titânio, na posição $2 q=38^{\circ}$. Uma análise sistemática entre $200^{\circ} \mathrm{C} \mathrm{e}$ $300^{\circ} \mathrm{C}$ confirma que a temperatura de transição entre o filme passivo e o filme cristalino de anatásia ocorre a aproximadamente $275^{\circ} \mathrm{C}$, porém nesta temperatura o filme de óxido é muito fino, e os picos observados correspondem ao substrato o material Ti-6Al-4V. Nas amostras de $400^{\circ} \mathrm{C}$ e $500^{\circ} \mathrm{C}$ observou-se comportamento idêntico, com as mesmas fases presentes na amostra de $300^{\circ} \mathrm{C}$, porém com uma redução moderada de intensidade dos picos de anatásia e titânio. $\mathrm{Na}$ amostra de $600^{\circ} \mathrm{C}$ iniciou o aparecimento de picos de rutilo e redução dos picos de anatásia e titânio. Picos de rutilo surgiram em várias posições, porém com intensidades moderadas. $\mathrm{Em} 650^{\circ} \mathrm{C}$ os picos de rutilo tornaramse mais evidentes, com intensidades elevadas, enquanto as fases de anatásia e titânio regrediram ainda mais em termos de intensidade. A $700^{\circ} \mathrm{C}$ observou-se ausência total de anatásia, alguns picos dispersos de titânio, e predominância da fase rutilo. Isto indica que em torno de $700^{\circ} \mathrm{C}$ a anatásia já não é mais estável, convertendo-se para rutilo, que passa a ser o único óxido estável. O filme de óxido tende a ser bastante efetivo contra corrosão até $650^{\circ} \mathrm{C}$. A partir desta temperatura, o crescimento do filme de óxido é controlado pela difusão. 
Figura 1: Espectro DRX das superfícies oxidadas por 5 horas em ar ambiente ( $T=$ titânio, $R=$ rutilo, $A=$ anatásia)

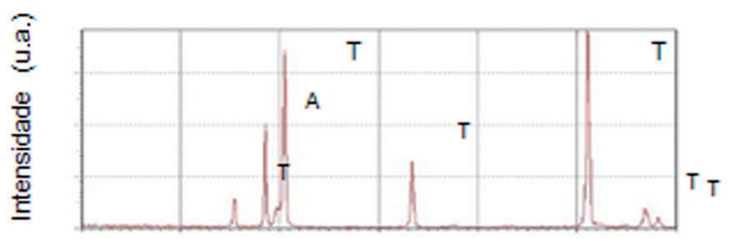

$300^{\circ} \mathrm{C}$

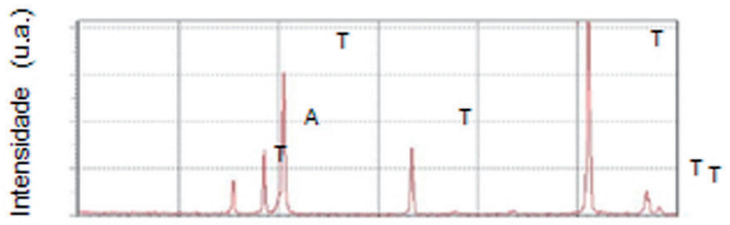

$400^{\circ} \mathrm{C}$

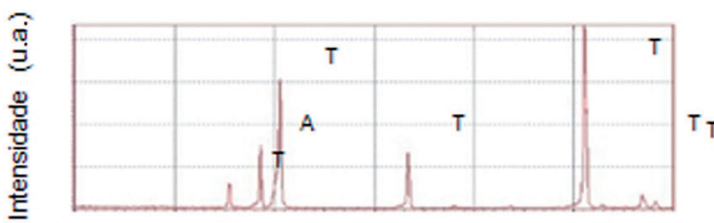

$500^{\circ} \mathrm{C}$

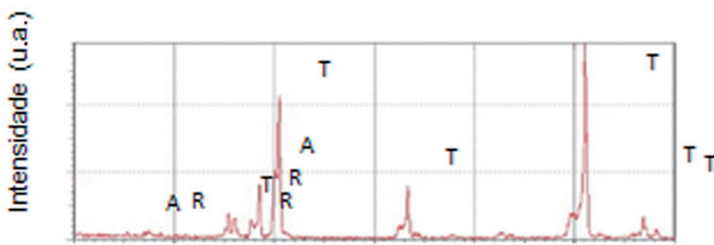

$600^{\circ} \mathrm{C}$

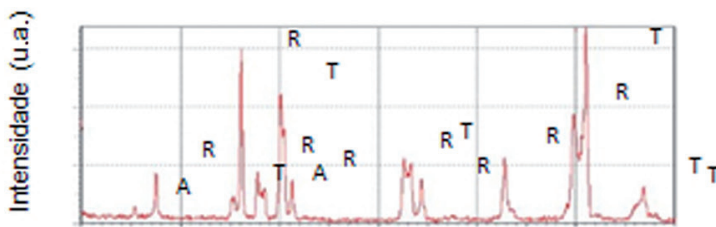

$650^{\circ} \mathrm{C}$

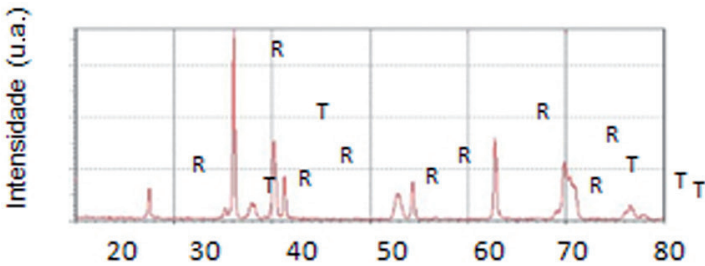

$700^{\circ} \mathrm{C}$

$2 \theta$ (graus)

Fonte: Da autora 


\subsection{Rugosidade Superficial}

A rugosidade média das superfícies das amostras antes e após a oxidação é apresentada na tabela 1. A figura 2 mostra que um aumento significativo de rugosidade ocorreu nas amostras oxidadas acima de $600^{\circ} \mathrm{C}$.

Tabela 1: Rugosidade superficial antes e após oxidação térmica de 5 horas em ar ambiente

\begin{tabular}{c|c|c}
\hline \multicolumn{3}{c}{ RUGOSIDADE SUPERFICIAL - Ra $(\mu \mathrm{m})$} \\
\hline Temperatura $\left({ }^{\circ} \mathrm{C}\right)$ & Antes Oxidação Térmica & Após Oxidação Térmica \\
$300^{\circ} \mathrm{C}$ & $0,06 \pm 0,01$ & $0,06 \pm 0,01$ \\
$400^{\circ} \mathrm{C}$ & $0,05 \pm 0,01$ & $0,05 \pm 0,01$ \\
$500^{\circ} \mathrm{C}$ & $0,05 \pm 0,01$ & $0,05 \pm 0,01$ \\
$600^{\circ} \mathrm{C}$ & $0,06 \pm 0,01$ & $0,07 \pm 0,02$ \\
$650^{\circ} \mathrm{C}$ & $0,07 \pm 0,01$ & $0,09 \pm 0,01$ \\
$700^{\circ} \mathrm{C}$ & $0,06 \pm 0,01$ & $0,13 \pm 0,01$ \\
\hline
\end{tabular}

Fonte: Da autora

Figura 2: Representação gráfica da evolução da rugosidade superficial

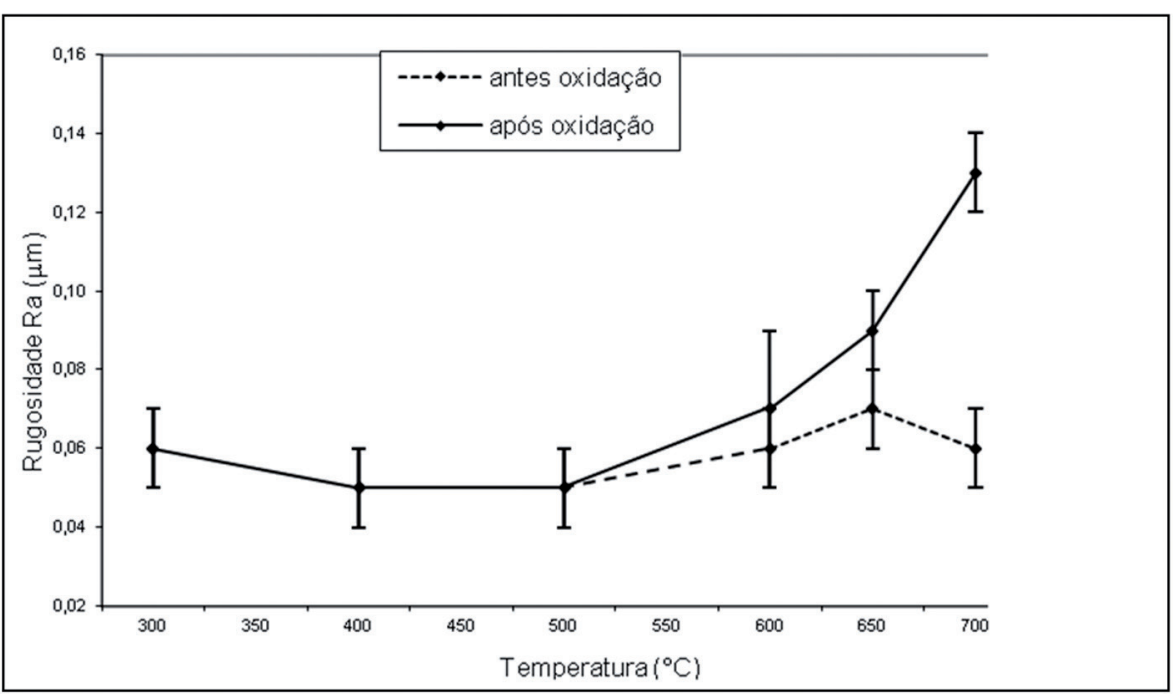

Fonte: Da autora

\subsection{Ensaios de Dureza}

A figura 3 indica os valores dureza das superfícies oxidadas. A dureza aumentou significativamente nas amostras oxidadas acima de $600^{\circ} \mathrm{C}$, alcançando maior valor $(8660$ $\mathrm{MPa}$ ) na amostra oxidada a $650^{\circ} \mathrm{C}$. Até $500^{\circ} \mathrm{C}$ a espessura dos filmes de óxido são muito 
finos, e a penetração efetuada na superfície indica a dureza do substrato (metal). Na amostra oxidada a $600^{\circ} \mathrm{C}$, a dureza provavelmente apresentou um valor intermediário entre a superfície do metal e a do filme de óxido, região também denominada de interface. A tabela 2 apresenta os resultados obtidos do ensaio de microdureza Vickers.

Tabela 2: Valores obtidos no ensaio de Microdureza Vickers

\begin{tabular}{l|l|l}
\hline \multirow{2}{*}{ Temperatura $\left({ }^{\circ} \mathrm{C}\right)$} & HV (MPa) \\
\cline { 2 - 3 } & Carga 98,01 mN & Carga 980,1 mN \\
\hline 25 & $4020,79 \pm 224,06$ & $3344,61 \pm 60,11$ \\
\hline 300 & $3920,32 \pm 172,41$ & $3488,06 \pm 74,16$ \\
\hline 400 & $3979,31 \pm 218,71$ & $3603,78 \pm 139,95$ \\
\hline 500 & $4322,51 \pm 166,91$ & $3774,20 \pm 92,82$ \\
\hline 600 & $7340,09 \pm 377,12$ & $4284,02 \pm 148,45$ \\
\hline 650 & --- & $8660,71 \pm 712,11$ \\
\hline 700 & --- & $6477,38 \pm 1176,39$ \\
\hline
\end{tabular}

Fonte: Da autora

Para os ensaios de Microdureza Vickers com cargas de 98,01 mN e 980,1 mN, observouse que com a carga menor $(98,01 \mathrm{mN})$ a dureza foi um pouco superior do que com a carga de 980,1 mN. Este fato ocorreu devido ao encruamento superficial das amostras, uma vez que estas foram adquiridas na forma de chapas laminadas. Com a carga de 980,1 mN é muito provável que se tenha ultrapassado a região encruada, daí a obtenção de durezas um pouco inferiores, caracterizando o substrato. Com a carga de 98,01 mN, só foi possível efetuar medições de microdureza até a amostra de $600^{\circ} \mathrm{C}$, pois acima desta temperatura a superfície apresentou uma coloração muito escura, inviabilizando a leitura do ponto penetrado. Com carga de $980,1 \mathrm{mN}$ foi possível efetuar a leitura de todos os pontos penetrados, porém, em função da coloração escura da amostra, não havia nitidez suficiente para efetuar uma leitura confiável, o que justifica o elevado desvio padrão para estas amostras. 
Figura 3: Medidas de dureza na superfície dos filmes de óxidos formados, após $5 \mathrm{~h}$ de oxidação em ar ambiente

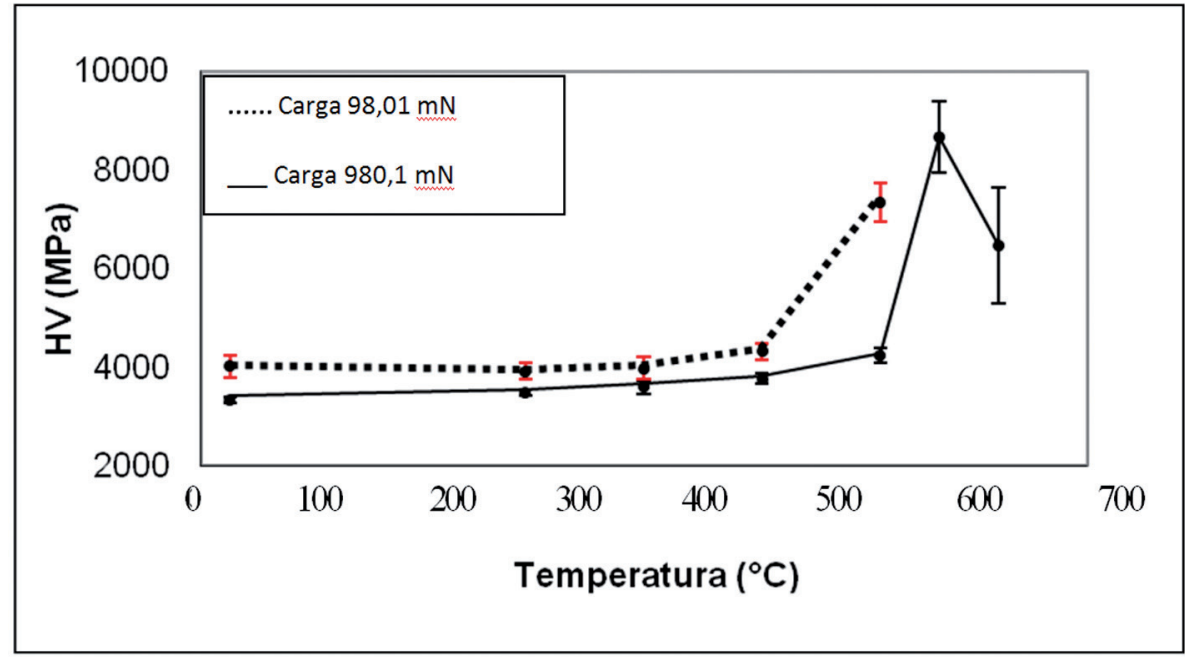

Fonte: Da autora

\subsection{Aderência HRC}

A aderência foi avaliada de forma qualitativa, nas superfícies das amostras oxidadas durante 5 horas em ar ambiente, de $300^{\circ} \mathrm{C}$ a $700^{\circ} \mathrm{C}$. A tabela 3 apresenta os resultados obtidos através da avaliação qualitativa da aderência, de acordo com a figura 4.

Nas amostras de $300^{\circ} \mathrm{C}$ e $400^{\circ} \mathrm{C}$, não houve sinais de fissuras, portanto não houve classificação de aderência. $\mathrm{Em} 500^{\circ} \mathrm{C}$ o filme de óxido apresentou fissuras radiais típicas de um filme dúctil, que pode ser relacionado com a classe $\mathrm{HF} 1$. A $600^{\circ} \mathrm{C}$, o filme de óxido apresentou uma densidade maior de fissuras radiais, porém foi identificado também como dúctil e com classificação entre $\mathrm{HF} 2$ e $\mathrm{HF} 3$. A $650^{\circ} \mathrm{C}$ observou-se finas e longas fissuras adjacentes ao contorno da impressão (ponto penetrado por Rockwell-C), com pequenas delaminações em torno da mesma. Estes tipos de fissuras representam aderência entre as classes HF 3 e HF 4, e também caracteriza-se como um filme dúctil. A $700^{\circ} \mathrm{C}$ o filme de óxido apresentou um comportamento bastante frágil, tornando inclusive visível macroscopicamente, quando foi aplicado o penetrador Rockwell-C, verificar pequenos fragmentos que se desprenderam devido ao rompimento do filme; porém na avaliação junto ao MEV comprovou-se esse fato através da visualização de grandes áreas de delaminação, e ficou então classificado entre HF 5 e HF 6. 
Figura 4: Classificação qualitativa de aderência HF1 a HF6

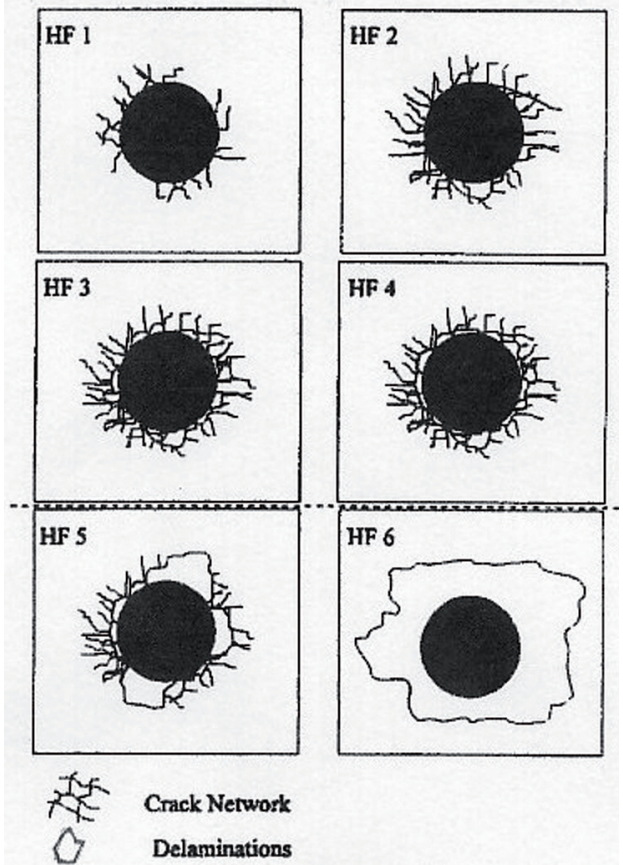

Fonte: Jehn; Reiners; Siegel, (1993)

Tabela 3: Classificação qualitativa para aderência dos filmes de óxido

\begin{tabular}{|l|l|}
\hline Temperatura $\left({ }^{\circ} \mathbf{C}\right)$ & Classificação HF \\
\hline 300 & ---- \\
\hline 400 & ---- \\
\hline 500 & 1 \\
\hline 600 & $2-3$ \\
\hline 650 & $3-4$ \\
\hline 700 & $5-6$ \\
\hline
\end{tabular}

Fonte: Da autora

A figura 5 mostra as fissuras e delaminações observadas a $600^{\circ} \mathrm{C}, 650^{\circ} \mathrm{C}$ e $700^{\circ} \mathrm{C}$. Acima de $650^{\circ} \mathrm{C}$ a taxa de oxidação foi acelerada devido aos efeitos térmicos e intrínsecos, formando um filme de óxido sob condições de maiores tensões, resultando em um filme 
de óxido fragilizado pela delaminação. Esse comportamento indica que o filme de óxido tem uma fraca aderência, ao contrário das amostras oxidadas entre $500^{\circ} \mathrm{C}$ e $650^{\circ} \mathrm{C}$, nas quais o filme de óxido apresentou uma boa aderência. Foram observadas as superfícies dos filmes de óxido, sem penetração Rockwell, e estas apresentaram superfícies livres de fissuras e uma rugosidade uniforme.

Figura 5: Delaminação e fissuras radiais às impressões produzidas por penetração Rockwell-C. Amostras com filme de óxido formado a $600^{\circ} \mathrm{C}$ (a), $650^{\circ} \mathrm{C}$ (b) e $700^{\circ} \mathrm{C}$ (c) e (d), após 5 horas de oxidação ao ar ambiente
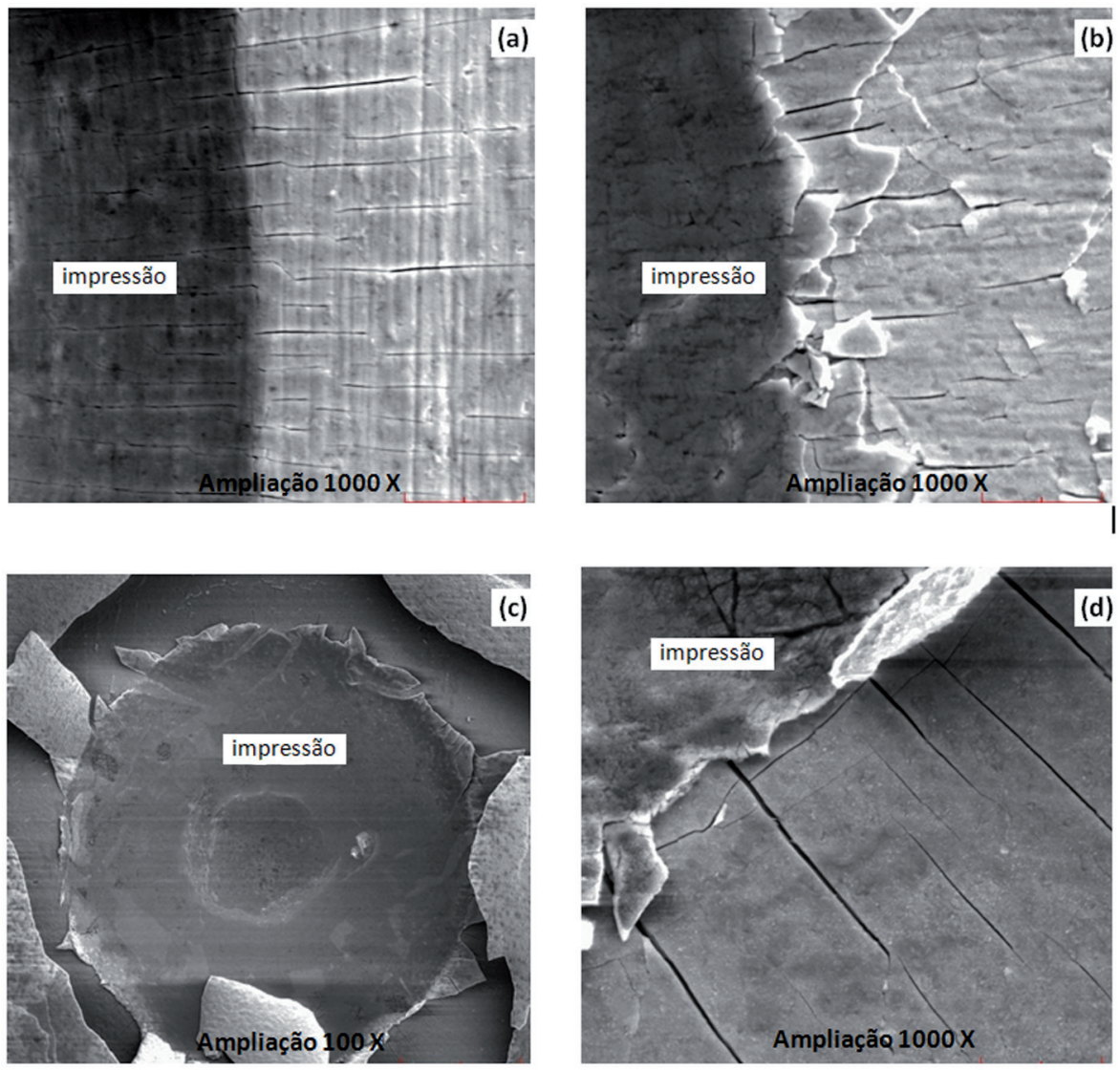

Fonte: Da autora

\section{CONCLUSÃO}

A liga de titânio Ti-6Al-4V comercial tem reação de oxidação de forma acelerada nos primeiros estágios do processo, levando à formação de filmes de óxido compostos de estrutura cristalina de $\mathrm{TiO} 2$ anatásia e rutilo. Após o período inicial, a cinética de oxidação ocorre de forma lenta até $600^{\circ} \mathrm{C}$ devido ao comportamento térmico. $O$ rutilo começou a surgir em torno de $600^{\circ} \mathrm{C}$ e é o único óxido estável a partir de $700^{\circ} \mathrm{C}$. Entre $600^{\circ} \mathrm{C}$ e $650^{\circ} \mathrm{C}$ o filme é composto das fases rutilo e anatásia simultaneamente. 
As análises de MEV mostraram que o filme de óxido formado na liga Ti-6Al-4V é isento de fissuras e possui uma rugosidade uniforme. $O$ teste de aderência Rockwell-C mostrou que o filme de óxido tem boa aderência nas amostras oxidadas entre $500^{\circ} \mathrm{C}$ e $650^{\circ} \mathrm{C}$. A $650^{\circ} \mathrm{C}$, a dureza superficial é de aproximadamente $8660 \mathrm{MPa}$. Estes resultados indicam que a liga de titânio comercial Ti-6Al-4V oxidada termicamente por 5 horas em ar ambiente, a temperatura de $650^{\circ} \mathrm{C}$, pode apresentar um bom desempenho biológico, tendo em vista que o filme de óxido apresenta boa aderência e uma morfologia de superfície que tende a aumentar a osseointegração através de fixação biomecânica.

\title{
INFLUENCE OF OXIDATION ON THE THERMAL PROPERTIES OF ALLOY TI 6AL 4V FOR APPLICATIONS IN PROSTHESIS - HEAT TREATMENT OF 5 HOURS AT AMBIENT AIR
}

\begin{abstract}
Titanium alloys are prominent among the materials for use in prostheses because they have high mechanical / weight resistance, good corrosion resistance and high biocompatibility. Among the titanium base alloys for biomedical applications, the main one is Ti-6Al-4V. However, recent research shows that the dissolution of Al and $\mathrm{V}$ may cause cytotoxic effects. To limit the dissolution of these elements was decided to form an oxide layer by thermal oxidation. This treatment also aimed to increase the surface roughness in order to improve bone-integration in case of implants. Therefore, this study aims at analyzing the influence of thermal oxidation on the surface properties of the Ti6Al-4V. Oxidation tests were carried out between 300 and $700{ }^{\circ} \mathrm{C}$ in air atmosphere for 5 hours. The oxides were evaluated by testing using $\mathrm{X}$-ray diffraction, scanning electron microscopy, Vickers hardness, adhesion by Rockwell-C hardness test, surface roughness and corrosion-wear tests. It was observed that the surface roughness increases with oxidation temperature. On the other hand, the hardness and corrosion resistance-wear achieve the best results for the samples oxidized at 600 and $650{ }^{\circ} \mathrm{C}$ respectively. The adhesion tests have shown that the oxide film has good adhesion at $600{ }^{\circ} \mathrm{C}$. Above this temperature the oxide film has low adhesion due to the presence of higher stresses in the film during formation.
\end{abstract}

Keywords: Ti-6Al-4V. Oxidation. Corrosion. Wear. Biomaterials. 


\section{REFERÊNCIAS}

BATISTA AVELAR, J.C. et al..Triode Plasma Nitriding and PVD Coating: A Successful PreTreatment Combination to Improve the Wear Resistance of DLC Coatings on Ti6Al4V Alloy. Elsevier - Surface \& Coatings Technology, [S.I.], vol.201, pp.4335-4340, 2006.

BUSER, D. et al.Influence of surface chacateristics on bone integration of titanium implants. J. Biomed. Mater. Res., Switzerland and Texas, vol. 25, pp. 889-902, 1991.

COCHRAN, D.L. et al. Response to unloaded and loades titanium implants with a sandblasted and acid-etched surface : a histometric study in the canine mandible. J. Biomed. Mater. Res., Switzerland and Texas, vol. 40, pp. 1-11, 1998.

GOTFREDSEN, K. et al. Anchorage of TiO2-blasted, HA-coated and machined implants: an experimental study with rabbits. J. Biomed. Mater. Res., Switzerland and Texas, vol.29, pp.1223-1231, 1995.

HEINKE, W. et al. Evaluation of PVD Nitride Coatings Using Impact, Scratch and Rockwell-C Adhesion Tests. Thin Solid Films, [S.I.], vol. 270, pp.431-438, 1995.

HENCH, L.L.; WILSON, J. Introduction to bioceramics. Singapore: Word Scientific Publishing Co. Pte Ltd., 1993. p. 1-15.

JEHN, H.; REINERS, G.; SIEGEL, N. (Eds.). DIN Fachbericht 39 - Characterisierung dünner Schichten. Berlin: Beuth Verlag, 1993.

LI, D. et al. Biomechanical comparison of the sandblasted and acid-etched and the machined and acid-etched titanium surface for dental implants. J. Biomed. Mater. Res., Switzerland and Texas, vol. 60, pp. 325-332, 2002.

MELLO ROCHA, G. M. et al. Efeito de Elementos Betagênicos nas Propriedades Mecânicas de Ligas de Titânio Tipo b Utilizadas em Implantes. BRAZILIAN CONFERENCE IN MATERIALS SCIENCE AND ENGINEERING, 16., 2004,Campina, SP. Proceedings... Campinas,SP, [s.n.], 2004.

NIINOMI, M. Mechanical Properties of Biomedical Titanium Alloys. Materials Science and Engineering, Japan, vol.243, pp.231-236, 1998.

OKAZAKI, Y.; GOTOH, E. Comparison of metal release from various metallic biomaterials in vivo.Biomaterials, Japan, vol. 26, pp.11-21, 2005.

ROCCI, A.; MARTIGNONI, M.; GOTLLOW, J. Immediate loading of Branemark system Ti unite and machined-surface implants in the posterior mandible : a randomized openended clinical trial. Clin. Implant Dent. Relat. Res., [S.I.], v. 5, p. 557-563, 2003.

SUL, Y.T. et al. Oxidized implants and their influence on the bone response. J. Mater. Sci. Mater. Med., Sweden-Korea-Germany, vol.12, pp.1025-1031, 2001. 
SUL, Y.T. et al. Resonance frequency and removal torque analysis of implants with turned an anodized surface oxides.Clin. Oral Implants Res, [S.I.],. vol. 13, pp. 252- 259, 2002.

SUL, Y.T. The significance of the surface properties of oxidized titanium to the bone response: special emphasis on potential biochemical bonding of oxidized titanium implant. Biomaterials, Sweden-Korea, vol. 24, pp. 3893-3907, 2003.

THOMSEN, P. et al. Structure of the interface between rabbit cortical bone and implants of gold, zirconium and titanium. J. Mater. Med., Sweden, vol. 8, pp.653-655, 1997.

WENNERBERG, A. et al. Histomorphometric and removal torque study of screw-shaped titanium implants with three different surface topographics. J. Clin. Oral Implants Res., [S.I.], vol. 6, pp. 24-30, 1996.

WENNERBERG, A. et al. Histomorphometric evaluation of screw-shaped implants each prepared with two surface roughnesses. Clin. Oral Implants Res., [S.I.],vol. 9, pp.11-19, 1998.

WENNERBERG, A. The importance of surface roughness for implants incorporation. Internations Journal of Machine Tools and Manufacture, Sweden, vol. 38, pp. 657-662, 1998.

ZIMMER, C. G. Análise de material de prótese de joelho explantada. In: CONGRESSO BRASILEIRO DE ENGENHARIA E CIÊNCIA DOS MATERIAIS, 17., 2006, Porto Alegre, RS. Anais eletrônicos... Porto Alegre, RS, Universidade Federal do Rio Grande do Sul, 2006. Disponível em: <http://www.environmentalchemistry.com/titanium.htm> Acesso em: 30 ago. 2012.

\section{SOBRE A AUTORA}

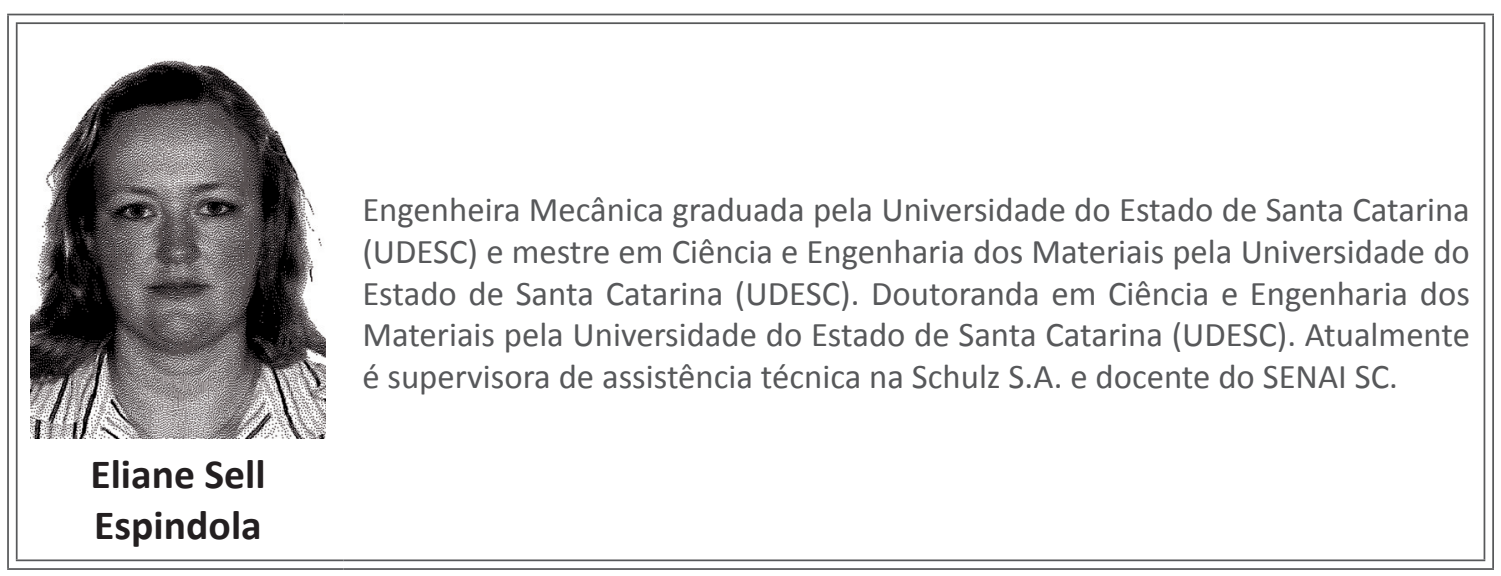

